\title{
A giant gastroenteric cyst associated with pectus excavatum and compression of the thoracic duct: A case report
}

\author{
Ismail Reisli, MD, ${ }^{a}$ Olgun Kadir Aribas, MD, ${ }^{\mathrm{b}}$ Yavuz Koksal, MD, ${ }^{\mathrm{a}}$ \\ Mustafa Cihat Avunduk, MD, ${ }^{\mathrm{c}}$ and Hasan Koc, MD, ${ }^{a}$ Meram-Konya, Turkey
}

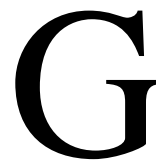

astroenteric cysts are unusual developmental cysts encountered in the posterior mediastinum of patients. They are usually lined by alimentary (squamous or enteric) epithelium and contain gastric mucosa and, rarely, pancreatic tissue. Gastroenteric cysts are usually associated with vertebral abnormalities and less often with complete situs inversus. ${ }^{1}$ We present a case of a gastroenteric cyst with pectus excavatum and compression of the thoracic duct.

\section{Clinical Summary}

A 6-month-old girl was admitted to the pediatric emergency unit with fever, cough, and dyspnea. On her past medical history, the presence of such a cyst in the right hemithorax had been demonstrated at the intrauterine 32nd week by means of ultrasonography (Figure 1, A), and the cyst had been aspirated to diminish its pressurizing effect. The patient was free of symptoms until 6 months of age. Physical examination on presentation revealed cyanosis, tachypnea, intercostal retractions, severe pectus excavatum, and diminished breath sounds and fine rales in the right hemithorax. The hemoglobin level of the patient was $10.5 \mathrm{~g} / \mathrm{dL}$, the leukocyte count was $19,900 / \mathrm{mm}^{3}$, the thrombocyte count was $840,000 / \mathrm{mm}^{3}$, the erythrocyte sedimentation rate was $12 \mathrm{~mm} / \mathrm{h}$, and the C-reactive protein level was $20.5 \mathrm{mg} / \mathrm{L}$. Arterial blood gas analyses revealed moderate hypoxemia and decreased oxygen saturation. Blood biochemical measurements were within normal limits. Posteroanterior and lateral chest roentgenograms showed homogenous opacity localized in the right hemithorax and pectus excavatum (Figure 1, $B$ and $C$ ). Thorax computed tomography revealed a cystic mass almost filling the right hemithorax (Figure $1, D)$. Through a right thoracotomy, a huge cyst, approximately 6 $\times 12 \mathrm{~cm}$ in size, was identified in the posterior mediastinum adjacent to the esophagus. In addition, the thoracic duct was deemed to be atrophic because of the compression of the cyst and

From the Departments of Pediatrics, ${ }^{\text {a }}$ Thoracic Surgery, ${ }^{\mathrm{b}}$ and Pathology, Meram Medical School of Selcuk University, Meram-Konya, Turkey.

Received for publication Jan 7, 2003; accepted for publication Jan 28, 2003

Address for reprints: Olgun Kadir Aribas, MD, Selcuk Universitesi Meram Tip Fakultesi, Gogus Cerrahisi Anabilim Dali, 42080, Meram-Konya, Turkey (E-mail: olgun@selcuk.edu.tr).

J Thorac Cardiovasc Surg 2003;126:584-5

Copyright (C) 2003 by The American Association for Thoracic Surgery $0022-5223 / 2003 \$ 30.00+0$

doi:10.1016/S0022-5223(03)00606-8 the proximal end. The distal ends of the thoracic duct were dilated, and therefore the cyst

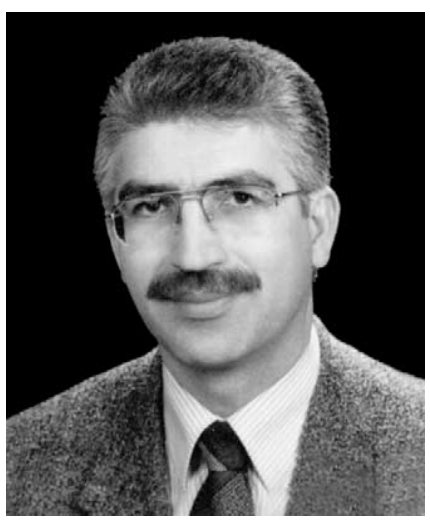

Dr Aribas was thought to be a cystic hygroma. The cyst was totally excised (Figure 2, A). On histologic examination, a gastric mucosa was seen within the cyst (Figure $2, B$ ). Nine months after surgical intervention, the patient remained well.

\section{Discussion}

Mediastinal cystic lesions constitute approximately $18 \%$ of all mediastinal lesions in children. Congenital foregut cysts of the mediastinum are extremely rare lesions. ${ }^{2}$ Sirivella and colleagues ${ }^{3}$ reported 4 enterogenous cysts among 20 patients with foregut cysts of the mediastinum. Eighty percent of the patients with foregut cysts were symptomatic in varying degrees of severity. Symptoms included dysphasia, pyrexia, cough, hematemesis, weight loss, retrosternal chest pain, and recurrent pneumonia. ${ }^{3}$ Our patient was symptomatic as well. Gastroenteric cysts are associated with vertebral abnormalities, such as scoliosis, anterior spina bifida, hemivertebrae, butterfly vertebrae, or vertebral fusions. We did not observe vertebral abnormalities; however, the patient had pectus excavatum.

Chest radiography, computed tomography, and magnetic resonance imaging are enough for the demonstration of a gastroenteric cyst. In addition, bronchoscopy and esophagoscopy might be helpful for the diagnosis. Barium swallow effectively pinpoints an extrinsic compression of the esophagus causing dysphasia. ${ }^{1-3}$ Congenital foregut cysts might be diagnosed on the basis of prenatal ultrasonography, as in our patient. ${ }^{4}$

Surgical excision of these cysts is indicated. Optimal timing for operation is dictated by the severity of symptoms. ${ }^{3}$ When an operation is not possible, bronchoscopic or thoracoscopic needle drainage might be alternative treatment procedures. However, these practices can cause severe complications, and in addition, the risk of malignant transformation will still remain. ${ }^{5}$ We preferred right thoracotomy for complete surgical removal of the cyst.

In this article we reported the first case, to our knowledge, of a patient presenting with a congenital gastroenteric cyst associated with pectus excavatum and compression of the thoracic duct. Although the cyst was aspirated during intrauterine life in an effort to decrease the compression of the cystic fluid, this procedure seemed to be unsuccessful because it resulted in atrophy after long-term compression of the thoracic duct. Therefore such cysts, whatever their size, should be removed as soon as possible before symptoms are evident. 

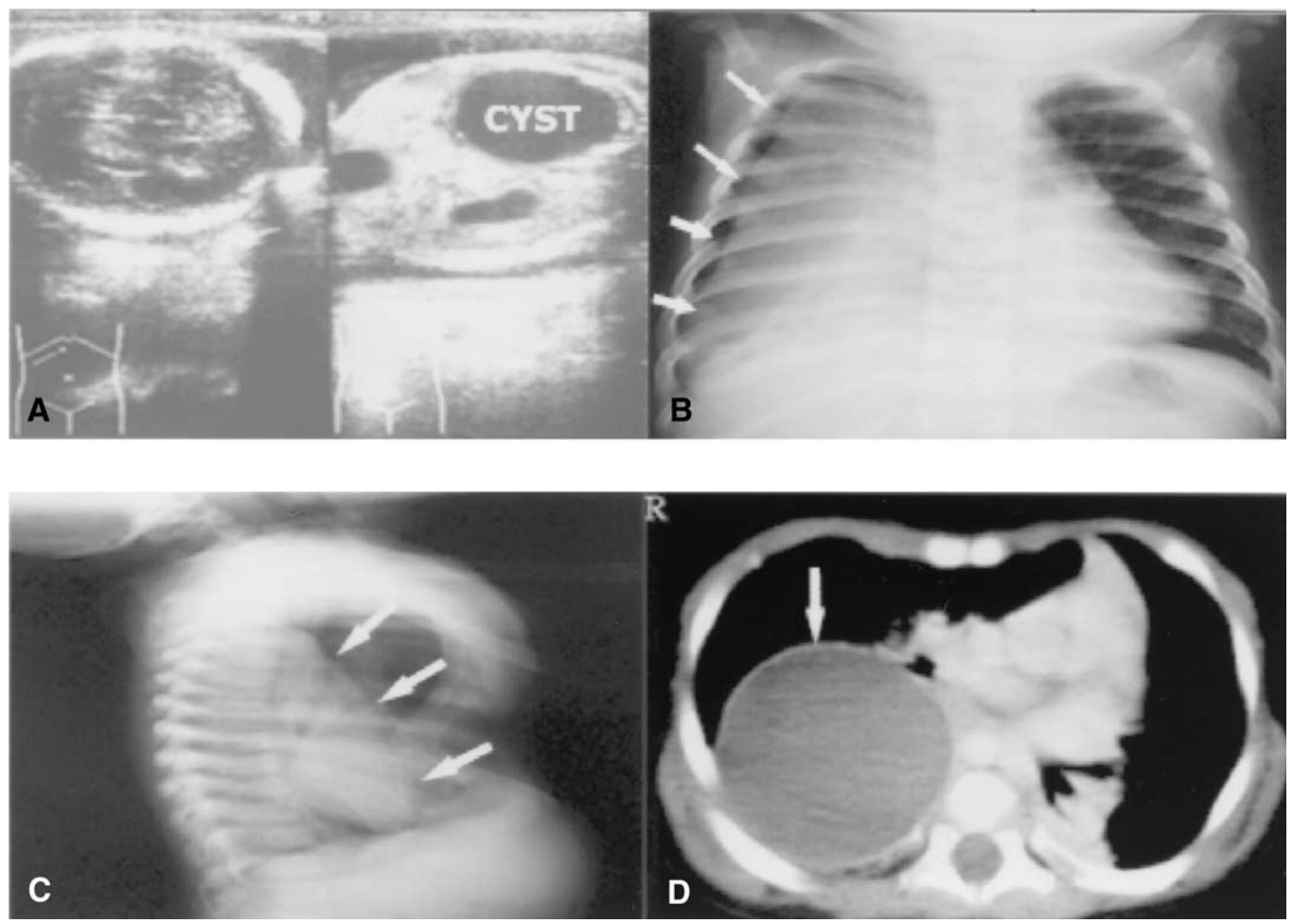

Figure 1. A, Prenatal ultrasonogram showing a cystic lesion in the right hemithorax. $B$, Posteroanterior chest radiograph. $C$, Pectus excavatum and the cyst were evident on the lateral chest radiograph. $D$, Chest computed tomographic scan showing a giant cystic mass

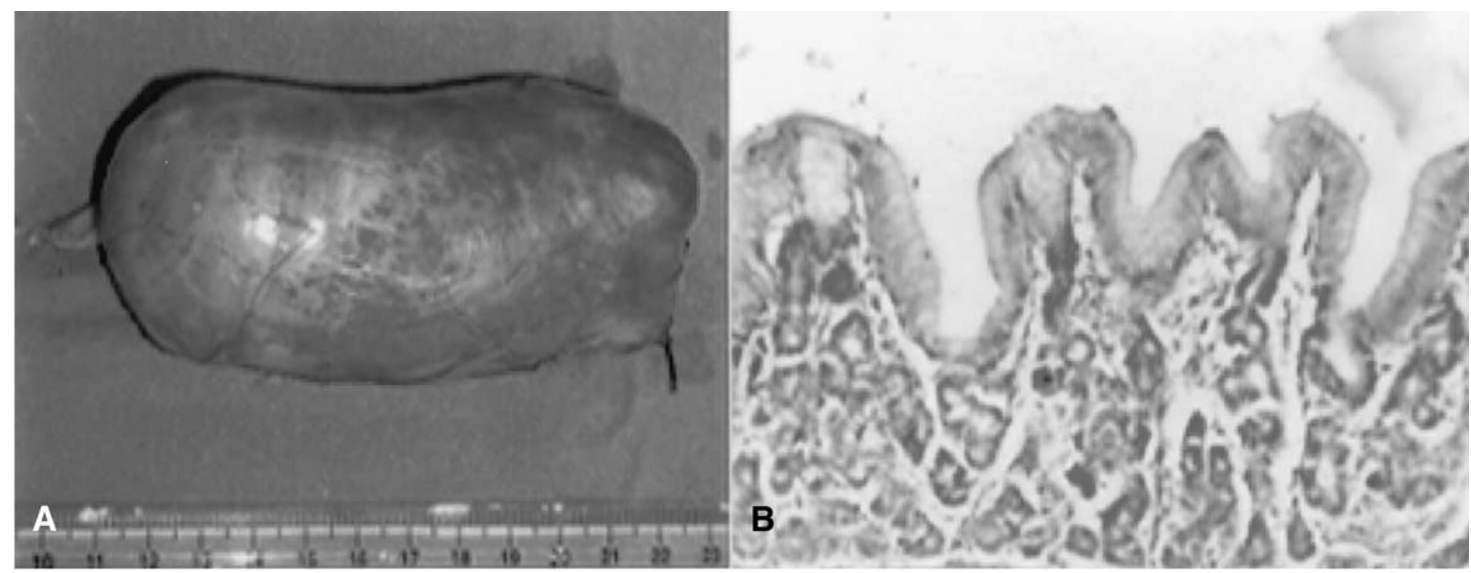

Figure 2. A, Macroscopic appearance of the totally removed giant cyst $(6 \times 12 \mathrm{~cm})$. $B$, Microscopic appearance of the cyst wall showing all layers of the gastrointestinal mucosa. (Hematoxylin and eosin staining, original magnification $40 \times$ ).

\section{References}

1. Akal M, Dikmen E, Sertçelik A, Dizbaysak S. Giant dumbbell-shaped gastroenteric cyst presenting with total situs inversus. Eur J Cardiothorac Surg. 2002;21:133-5.

2. Ochsner JL, Ochsner SF. Congenital cysts of the mediastinum: 20-year experience with 42 cases. Ann Surg. 1966;163:909-19.

3. Sirivella S, Ford WB, Zikria EA, Miller WH, Samadani SR, Sullivan
ME. Foregut cysts of the mediastinum results in 20 consecutive surgically treated cases. J Thorac Cardiovasc Surg. 1985;90:776-82.

4. dell'Agnola C, Tadini B, Mosca F, Colnaghi M, Wesley J. Advantages of prenatal diagnosis and early surgery for congenital cystic disease of the lung. J Perinat Med. 1996;24:621-31.

5. Smith SM, Young CS, Bishop AF. Adenocarcinoma of a foregut cyst. Detection with positron emission tomography. Am J Roentgenol. 1996; 167:1153-4. 